\title{
UPPER AND LOWER SOLUTIONS METHOD FOR IMPULSIVE DIFFERENTIAL INCLUSIONS WITH NONLINEAR BOUNDARY CONDITIONS
}

\author{
YAN LUO \\ School of Mathematics and Computing Science \\ Hunan University of Science and Technology \\ Xiangtan, Hunan, 411201, P.R. CHINA
}

ABSTRACT: This paper discusses the antiperiodic and nonlinear boundary problem for first-order impulsive differential inclusions. We establish the existence results by using Martelli's fixed point theorem with upper and lower solutions method.

AMS Subject Classification: 34A60, 34A37, 47H10

Key Words: impulsive differential inclusions, antiperiodic and nonlinear boundary value, fixed point theorem, lower and upper solution

Received: January 8, 2018; Accepted: April 1, 2018;

Published: April 10, 2018 doi: 10.12732/dsa.v27i2.11

Dynamic Publishers, Inc., Acad. Publishers, Ltd. https://acadsol.eu/dsa

\section{INTRODUCTION}

In this paper, we consider the first order impulsive differential inclusions with antiperiodic and nonlinear boundary conditions

$$
\left\{\begin{array}{l}
x^{\prime}(t) \in F(t, x(t)), t \in J^{\prime}, \\
\Delta x\left(t_{k}\right)=I_{k}\left(x\left(t_{k}\right)\right), k=1, \ldots, m, \\
g(x(0), x(T))=0,
\end{array}\right.
$$

where $J^{\prime}=J \backslash\left\{t_{1}, \ldots, t_{m}\right\}, J=[0, T], T>0,0<t_{1}<t_{2}<\cdots<t_{m}<T$, $\Delta x\left(t_{k}\right)=x\left(t_{k}^{+}\right)-x\left(t_{k}^{-}\right), x\left(t_{k}^{+}\right)=\lim _{\varepsilon \rightarrow 0^{+}} x\left(t_{k}+\varepsilon\right), x\left(t_{k}^{-}\right)=\lim _{\varepsilon \rightarrow 0^{+}} x\left(t_{k}-\varepsilon\right)$, $F: J \times R \rightarrow P(R)$ is a multivalued map, $P(R)$ is the family of all nonempty subsets of $R, I_{k} \in C(R, R)(k=1, \ldots, m)$, and $g: R^{2} \rightarrow R$ is a single valued map.

The theory of impulsive differential equations or inclusions has become an active area of investigation due to their applications in the fields of mechanics, electrical 
engineering, medicine biology, ecology, and so on, see $[5,6,12,18]$. Critical point theory plays a major role in discussing the existence of solutions for boundary problem for impulsive differential inclusions, see $[9,10,11,16,17]$. There are many other methods such as in $[1,3,7]$. In [4], authors considered periodic boundary conditions $g(x, y)=x-y$, i.e., $x(0)=x(T)$. Those results are applicable in some important cases. However, they are not valid for antiperiodic boundary conditions, for example, $x(0)=-x(T)$, which corresponds to $g(x, y)=x+y$. Note that in this case $g$ is nondecreasing in the second variable, and hence the results are not applicable. To the author's best knowledge, there is no paper discussing such boundary problem for impulsive differential inclusions.

Motivated by the above mentioned works, the aim of this paper is to study the existence of solutions for antiperiodic and nonlinear boundary problem (1.1) by Martelli's fixed point theorem with upper and lower solutions method. The rest of the paper is organized as follows. In Section 2, we introduce briefly some notations and necessary preliminaries. In Section 3, we prove the existence results of solutions for system (1.1).

\section{PRELIMINARIES}

In the paper, we introduce some notations, definitions and preliminary facts, which are useful for the development of this paper.

Let $X$ be a Banach space, $Z$ be a subset of $X$. We denote $P(X)=\{Z \subset X \mid Z \neq$ $\emptyset\}, P_{c v}(X)=\{Z \subset P(X) \mid Z$ is convex $\}, P_{c p}(X)=\{Z \subset P(X) \mid Z$ is compact $\}$, $P_{c v, c p}(X)=P_{c v}(X) \cap P_{c p}(X)$, and so forth. For example, let $X=R$, we have notations $P(R), P_{c v}(R), P_{c p}(R)$ and $P_{c v, c p}(R)$. Let

$$
L^{1}(J, R)=\{x: J \rightarrow R|| x \mid: J \rightarrow[0,+\infty) \text { is Lebesgue integrable }\},
$$

then $L^{1}(J, R)$ is a Banach space with the norm $\|x\|_{L^{1}}=\int_{0}^{T}|x(t)| d t$.

$$
\begin{array}{r}
P C(J, R)=\left\{x: J \rightarrow R \mid x(t) \text { is continuous everywhere except for some } t_{k}\right. \text {, at which } \\
\left.x\left(t_{k}^{-}\right), x\left(t_{k}^{+}\right) \text {exist and } x\left(t_{k}^{-}\right)=x\left(t_{k}\right), k=1, \ldots, m\right\},
\end{array}
$$

which is a Banach space with the norm $\|x\|_{P C}=\sup \{|x(t)|: t \in J\}$.

$A C(J, R)$ is the space of all absolutely continuous functions $x: J \rightarrow R$.

Definition 1. Throughout this paper, the multivalued map $F: J \times R \rightarrow P(R)$ is said to be $L^{1}$-Carathéodory if

(i) $t \rightarrow F(t, x)$ is measurable for each $x \in R$, 
(ii) $x \rightarrow F(t, x)$ is upper semicontinuous on $R$ for almost all $t \in J$,

(iii) for each $\rho>0$, there exists $\varphi_{\rho} \in L^{1}(J,[0,+\infty))$ such that

$$
\|F(t, x)\|_{P(R)}=\sup \{|v|: v \in F(t, x)\} \leq \varphi_{\rho}(t), \forall|x| \leq \rho \text {, a.e. } t \in J .
$$

Definition 2. The function $\alpha, \beta \in P C(J, R) \cap A C\left(J^{\prime}, R\right)$ are said to be related lower and upper solutions for the antiperiodic problem (1.1) if there exist $v_{1}, v_{2} \in L^{1}(J, R)$ such that

$$
\left\{\begin{array}{l}
v_{1}(t) \in F(t, \alpha(t)), \text { a.e. } t \in J \\
\alpha^{\prime}(t) \leq v_{1}(t), \text { a.e. } t \in J^{\prime} \\
\Delta \alpha\left(t_{k}\right) \leq I_{k}\left(\alpha\left(t_{k}\right)\right), k=1, \ldots, m \\
g(\alpha(0), \beta(T)) \leq 0
\end{array}\right.
$$

and

$$
\left\{\begin{array}{l}
v_{2}(t) \in F(t, \beta(t)), \text { a.e. } t \in J \\
\beta^{\prime}(t) \geq v_{2}(t), \text { a.e. } t \in J^{\prime} \\
\Delta \beta\left(t_{k}\right) \geq I_{k}\left(\beta\left(t_{k}\right)\right), k=1, \ldots, m \\
g(\beta(0), \alpha(T)) \geq 0 .
\end{array}\right.
$$

Definition 3. A function $x \in P C(J, R) \cap A C\left(J^{\prime}, R\right)$ is said to be a solution of (1.1) if there exists a function $v \in L^{1}(J, R)$ such that $v(t) \in F(t, x(t))$ a.e. on $J, x^{\prime}(t)=v(t)$ a.e. on $J^{\prime}, \Delta x\left(t_{k}\right)=I_{k}\left(x\left(t_{k}\right)\right), k=1, \ldots, m$, and $g(x(0), x(T))=0$.

Lemma 4. (see [14]) Let $X$ be a Banach space, and $F: J \times X \rightarrow P_{c v, c p}(X)$ be a $L^{1}$-Carathéodory multivalued map with

$$
S_{F, x}=\left\{f \in L^{1}(J, X) \mid f(t) \in F(t, x(t)) \text { for a.e. } t \in J\right\} \neq \emptyset,
$$

and let $\Gamma: L^{1}(J, X) \rightarrow C(J, X)$ be a linear continuous mapping, then the operator

$$
\Gamma \circ S_{F}: C(J, X) \rightarrow P_{c v, c p}(C(J, X)), u \mapsto\left(\Gamma \circ S_{F}\right)(x):=\Gamma\left(S_{F, x}\right)
$$

is a closed graph operator in $C(J, X) \times C(J, X)$.

Lemma 5. (Martelli's fixed point theorem [15]) Let $X$ be a Banach space and $G: X \rightarrow P_{c v, c p}(X)$ be an upper semicontinuous and condensing map. If the set $\Re=\{x \in X: \lambda x \in G(x)$ for some $\lambda>1\}$ is bounded, then $G$ has a fixed point.

Remark 6. (i) If multivalued map $F$ is completely continuous with nonempty compact values, then $F$ is upper semicontinuous if and only if $F$ has a closed graph (i.e., $x_{n} \rightarrow x^{*}, y_{n} \rightarrow y^{*}, y_{n} \in F\left(x_{n}\right)$ imply $y^{*} \in F\left(x^{*}\right)$ ).

(ii) If multivalued map $F$ is completely continuous, then $F$ is condensing. For general information the reader can see [13].

Let $J_{0}=\left[0, t_{1}\right], J_{k}=\left(t_{k}, t_{k+1}\right], k=1, \ldots, m, t_{m+1}=T$. 
Definition 7. (See [2]) A family of functions $S$ is said to be quasiequicontinuous on $J$, if for every $\varepsilon>0$ there exists $\delta>0$ such that if $x \in S, k=0,1, \ldots, m$, then

$$
\left\|x\left(t_{1}\right)-x\left(t_{2}\right)\right\|<\varepsilon, \forall t_{1}, t_{2} \in J_{k} \text { and }\left|t_{1}-t_{2}\right|<\delta .
$$

Lemma 8. (Compactness Criterion, see [2]) The set $S \in P C\left(J, R^{n}\right)$ is relatively compact if and only if

(i) $S$ is bounded, i.e., $\|x\|<c$ for each $x \in S$ and some $c>0$,

(ii) $S$ is quasiequicontinuous on $J$.

Definition 9. Let $X$ be a Banach space, a multivalued map $F$ is said to be completely continuous if $F(U)$ is relatively compact for every bounded subset $U \subseteq X$.

\section{MAIN RESULT}

Theorem 10. Assume the following conditions hold.

(H1) $F: J \times R \rightarrow P_{c v, c p}(R)$ is an $L^{1}$-Carathéodory multivalued map.

(H2) Functions $\alpha, \beta \in P C(J, R) \cap A C\left(J^{\prime}, R\right)$ are related lower and upper solutions of problem (1.1) such that $\alpha(t) \leq \beta(t), t \in J$.

(H3) $I_{k} \in C(R, R), k=1, \ldots, m$.

(H4) $g$ is a continuous single-valued map in $(x, y) \in[\alpha(0), \beta(0)] \times[\alpha(T), \beta(T)]$, and nondecreasing in $y \in[\alpha(T), \beta(T)]$.

Then the system (1.1) has at least one solution $x$ such that $\alpha(t) \leq x(t) \leq \beta(t)$ for all $t \in J$.

Proof. We transform (1.1) into a fixed point problem. Consider the modified problem

$$
\left\{\begin{array}{l}
x^{\prime}(t)+x(t) \in F_{1}(t, x(t)), t \in J^{\prime} \\
\Delta x\left(t_{k}\right)=I_{k}\left(\tau\left(t_{k}, x\left(t_{k}\right)\right)\right), k=1, \ldots, m \\
x(0)=\tau(0, x(0)-g(\tau(0, x), \tau(T, x))),
\end{array}\right.
$$

where $F_{1}(t, x)=F(t, \tau(t, x))+\tau(t, x), \tau: C(J, R) \rightarrow C(J, R)$ is defined by

$$
\tau(t, x)= \begin{cases}\beta(t), & x(t)>\beta(t) \\ x(t), & \alpha(t) \leq x(t) \leq \beta(t) \\ \alpha(t), & x(t)<\alpha(t)\end{cases}
$$

Evidently, if $x$ is a solution of (3.1), $\alpha(t) \leq x(t) \leq \beta(t)$, and $\alpha(0) \leq x(0)-$ $g(\tau(0, x), \tau(T, x)) \leq \beta(0)$, then $x$ is a solution of $(1.1)$.

By straightforward calculation, we have that a solution of (3.1) is a fixed point of the operator $N: P C(J, R) \rightarrow P(P C(J, R))$ defined by 


$$
\begin{aligned}
N(x)=\left\{h \in P C(J, R): h(t)=x(0)+\int_{0}^{t}\right. & {[v(s)+\tau(s, x)-x(s)] d s } \\
& \left.+\sum_{0<t_{k}<t} I_{k}\left(\tau\left(t_{k}, x\left(t_{k}\right)\right)\right), v \in \widetilde{S}_{F, x}\right\},
\end{aligned}
$$

where

$$
\begin{gathered}
\widetilde{S}_{F, x}=\left\{v \in S_{F, \tau(t, x)}: v(t) \geq v_{1}(t) \text { a.e.on } A_{1}, v(t) \leq v_{2}(t) \text { a.e. on } A_{2}\right\} \\
S_{F, \tau(t, x)}=\left\{v \in L^{1}(J, R): v(t) \in F(t, \tau(t, x)) \text { for a.e. } t \in J\right\} \\
A_{1}=\{t \in J: x(t)<\alpha(t) \leq \beta(t)\}, A_{2}=\{t \in J: \alpha(t) \leq \beta(t)<x(t)\} .
\end{gathered}
$$

Note that for each $x \in C(J, R), S_{F, x}$ is nonempty (see [14]), so $\widetilde{S}_{F, x}$ is nonempty.

Next we will show that $N$ has a fixed point by applying Lemma 5 . The proof will be given in several steps. We first show that $N$ is a completely continuous multivalued map, upper semicontinuous with convex closed values.

Step 1. $N(x)$ is convex for each $x \in P C(J, R)$.

Indeed, if $h_{1}, h_{2}$ belong to $N(x)$, then there exist $\bar{v}_{1}, \bar{v}_{2} \in \widetilde{S}_{F, x}$ such that

$$
h_{i}(t)=x(0)+\int_{0}^{t}\left[\bar{v}_{i}(s)+\tau(s, x)-x(s)\right] d s+\sum_{0<t_{k}<t} I_{k}\left(\tau\left(t_{k}, x\left(t_{k}\right)\right)\right),
$$

$i=1,2$. Let $0 \leq l \leq 1$, then for each $t \in J$, we have

$$
\begin{aligned}
{\left[l h_{1}+(1-l) h_{2}\right](t) } & =x(0)+\sum_{0<t_{k}<t} I_{k}\left(\tau\left(t_{k}, x\left(t_{k}\right)\right)\right) \\
& +\int_{0}^{t}\left[l \bar{v}_{1}(s)+(1-l) \bar{v}_{2}(s)+\tau(s, x)-x(s)\right] d s .
\end{aligned}
$$

Since $\widetilde{S}_{F, x}$ is convex (because $F$ has convex values in (H1)), then $l h_{1}+(1-l) h_{2} \in N(x)$, so $N(x)$ is convex.

Step 2. $N$ is completely continuous.

Firstly, we show that $N$ maps bounded sets into bounded sets in $P C(J, R)$. Let $q$ be a positive constant, $B_{q}=\left\{x \in P C(J, R):\|x\|_{P C}<q\right\}$ be a bounded set, and $x \in B_{q}$. Then for each $h \in N(x)$, there exists $v \in \widetilde{S}_{F, x}$ such that

$$
h(t)=x(0)+\int_{0}^{t}[v(s)+\tau(s, x)-x(s)] d s+\sum_{0<t_{k}<t} I_{k}\left(\tau\left(t_{k}, x\left(t_{k}\right)\right)\right) .
$$

Note the boundary condition of (3.1) and the definition of $\tau$, we have

$$
\begin{gathered}
\alpha(0) \leq x(0) \leq \beta(0), \\
\alpha(t) \leq \tau(t, x) \leq \beta(t) .
\end{gathered}
$$


Let $\rho_{1}=\max \left(q, \sup _{t \in J}|\alpha(t)|, \sup _{t \in J}|\beta(t)|\right)$, then $|\tau(t, x)| \leq \rho_{1}$. From (H1), there exists $\varphi_{\rho_{1}} \in L^{1}(J,[0,+\infty))$ such that

$$
\sup \{|v|: v \in F(t, \tau(t, x))\} \leq \varphi_{\rho_{1}}(t) .
$$

If $x \in B_{q}$, then there exist $c_{k}>0, k=1, \ldots, m$ such that

$$
\left|I_{k}\left(\tau\left(t_{k}, x\left(t_{k}\right)\right)\right)\right| \leq c_{k}
$$

since $I_{k}$ are continuous in (H3) and (3.4). So, with (3.3) and (3.5), we have

$$
\begin{aligned}
|h(t)| & \leq|x(0)|+\int_{0}^{T}[|v(s)|+|\tau(s, x)|+|x(s)|] d s+\sum_{0<t_{k}<t}\left|I_{k}\left(\tau\left(t_{k}, x\left(t_{k}\right)\right)\right)\right| \\
& \leq \max (|\alpha(0)|,|\beta(0)|)+\left\|\varphi_{\rho_{1}}\right\|_{L^{1}}+T\left(\rho_{1}+q\right)+\sum_{k=1}^{m} c_{k}:=K,
\end{aligned}
$$

then $\|N(x)\|_{P C} \leq K$.

Secondly, we prove that $N$ maps bounded sets into quasiequicontinuous sets of $P C(J, R)$. Let $u_{1}, u_{2} \in J_{k}, k=0,1, \ldots, m, u_{1}<u_{2}, x \in B_{q}$ and $h \in N(x)$. Then,

$$
\left|h\left(u_{2}\right)-h\left(u_{1}\right)\right| \leq \int_{u_{1}}^{u_{2}} \varphi_{\rho_{1}}(s) d s+\left(u_{2}-u_{1}\right)\left(\rho_{1}+q\right)+\sum_{u_{1}<t_{k}<u_{2}} c_{k} .
$$

As $u_{2} \rightarrow u_{1}$, the right-hand side of the above inequality tends to zero. This proves that $N\left(B_{q}\right)$ is quasiequicontinuous. By Lemma $8, N$ is completely continuous, and therefore a condensing map.

Step 3. $N$ has a closed graph.

Let $x_{n} \rightarrow x^{*}, h_{n} \in N\left(x_{n}\right)$, and $h_{n} \rightarrow h^{*}$. We will prove that $h^{*} \in N\left(x^{*}\right)$. $h_{n} \in N\left(x_{n}\right)$ means that there exists $v_{n} \in \widetilde{S}_{F, x_{n}}$ such that

$$
h_{n}(t)=x_{n}(0)+\int_{0}^{t}\left[v_{n}(s)+\tau\left(s, x_{n}\right)-x_{n}(s)\right] d s+\sum_{0<t_{k}<t} I_{k}\left(\tau\left(t_{k}, x_{n}\left(t_{k}\right)\right)\right) .
$$

Next we need prove that there exists $v^{*} \in \widetilde{S}_{F, x^{*}}$ such that for each $t \in J$,

$$
h^{*}(t)=x^{*}(0)+\int_{0}^{t}\left[v^{*}(s)+\tau\left(s, x^{*}\right)-x^{*}(s)\right] d s+\sum_{0<t_{k}<t} I_{k}\left(\tau\left(t_{k}, x^{*}\left(t_{k}\right)\right)\right) .
$$

Since $x_{n} \rightarrow x^{*}, h_{n} \rightarrow h^{*}, \tau$ and $I_{k}(k=1,2, \ldots, m)$ are continuous, we have

$$
\begin{aligned}
& \| h_{n}(t)-x_{n}(0)-\int_{0}^{t}\left[\tau\left(s, x_{n}\right)-x_{n}(s)\right] d s-\sum_{0<t_{k}<t} I_{k}\left(\tau\left(t_{k}, x_{n}\left(t_{k}\right)\right)\right)- \\
& {\left[h^{*}(t)-x^{*}(0)-\int_{0}^{t}\left[\tau\left(s, x^{*}\right)-x^{*}(s)\right] d s-\sum_{0<t_{k}<t} I_{k}\left(\tau\left(t_{k}, x^{*}\left(t_{k}\right)\right)\right)\right] \|_{P C}} \\
& \rightarrow 0
\end{aligned}
$$


as $n \rightarrow \infty$. Consider the linear and continuous operator $\Gamma: L^{1}(J, R) \rightarrow C(J, R)$,

$$
v \mapsto \Gamma(v)(t)=\int_{0}^{t} v(s) d s .
$$

From Lemma $4, \Gamma \circ \widetilde{S_{F}}$ is a closed graph operator. Moreover,

$$
h_{n}(t)-x_{n}(0)-\int_{0}^{t}\left[\tau\left(s, x_{n}\right)-x_{n}(s)\right] d s-\sum_{0<t_{k}<t} I_{k}\left(\tau\left(t_{k}, x_{n}\left(t_{k}\right)\right)\right) \in \Gamma\left(\widetilde{S}_{F, x_{n}}\right) .
$$

Since $x_{n} \rightarrow x^{*},(3.6)$ and (3.7), there exists $v^{*} \in \widetilde{S}_{F, x^{*}}$ satisfying

$$
h^{*}(t)-x^{*}(0)-\int_{0}^{t}\left[\tau\left(s, x^{*}\right)-x^{*}(s)\right] d s-\sum_{0<t_{k}<t} I_{k}\left(\tau\left(t_{k}, x^{*}\left(t_{k}\right)\right)\right)=\int_{0}^{t} v^{*}(s) d s .
$$

As a consequence of Steps 1 to $3, N$ is a completely continuous multivalued map, upper semicontinuous with convex closed values.

Step 4. The set $\Re=\{x \in P C(J, R): \lambda x \in N(x)$ for some $\lambda>1\}$ is bounded.

Let $x \in \Re$, then $\lambda x \in N(x)$ for some $\lambda>1$. Thus, for each $t \in J$,

$$
x(t)=\lambda^{-1}\left[x(0)+\int_{0}^{t}[v(s)+\tau(s, x)-x(s)] d s+\sum_{0<t_{k}<t} I_{k}\left(\tau\left(t_{k}, x\left(t_{k}\right)\right)\right)\right],
$$

for some $v \in \widetilde{S}_{F, x}$. Let $\rho_{2}=\max \left(\sup _{t \in J}|\alpha(t)|, \sup _{t \in J}|\beta(t)|\right)$, it follows from (3.4) that $|\tau(t, x)| \leq \rho_{2}$. From (H1), there exists $\varphi_{\rho_{2}} \in L^{1}(J,[0,+\infty))$ such that

$$
\sup \{|v|: v \in F(t, \tau(t, x))\} \leq \varphi_{\rho_{2}}(t) .
$$

Since $I_{k}$ are continuous in (H3) and (3.4), there exist $c_{k}^{\prime}>0, k=1, \ldots, m$ such that $\left|I_{k}\left(\tau\left(t_{k}, x\left(t_{k}\right)\right)\right)\right| \leq c_{k}^{\prime}$. So, with (3.3) and (3.8), for each $t \in J$ we have

$$
\begin{aligned}
|x(t)| \leq & |x(0)|+\int_{0}^{t}[|v(s)|+|\tau(s, x)|+|x(s)|] d s \\
& +\sum_{0<t_{k}<t}\left|I_{k}\left(\tau\left(t_{k}, x\left(t_{k}\right)\right)\right)\right| \\
\leq & \max (|\alpha(0)|,|\beta(0)|)+\left\|\varphi_{\rho_{2}}\right\|_{L^{1}}+T \rho_{2} \\
& +\int_{0}^{t}|x(s)| d s+\sum_{k=1}^{m} c_{k}^{\prime} .
\end{aligned}
$$

Set

$$
K_{0}=\max (|\alpha(0)|,|\beta(0)|)+\left\|\varphi_{\rho_{2}}\right\|_{L^{1}}+T \rho_{2}+\sum_{k=1}^{m} c_{k}^{\prime} .
$$

Using Gronwall's lemma (see [8], page 36), for each $t \in J$, we have

$$
|x(t)| \leq K_{0} e^{t} .
$$


So,

$$
\|x\|_{P C} \leq K_{0} e^{T}
$$

This shows that the set $\Re$ is bounded. As a consequence of Lemma 5 , we deduce that $N$ has a fixed point, which is a solution of problem (3.1).

Step 5. The solution $x$ of (3.1) satisfies

$$
\alpha(t) \leq x(t) \leq \beta(t), t \in J
$$

and

$$
\alpha(0) \leq x(0)-g(\tau(0, x), \tau(T, x)) \leq \beta(0) .
$$

We firstly prove (3.9). Let $x$ be a solution of (3.1), we prove that $x(t) \leq \beta(t)$, for all $t \in J$. Suppose that $x-\beta$ attains a positive maximum on $J$ at $s_{0}$. As (3.3), we consider the only possible case $s_{0} \in(0, T]$. Then there exists $s_{1} \in\left(0, s_{0}\right)$ and $s_{1} \neq t_{k}(k=1,2, \ldots, m)$ such that

$$
0<x(t)-\beta(t) \leq x\left(s_{0}\right)-\beta\left(s_{0}\right), t \in\left[s_{1}, s_{0}\right]
$$

So, $\tau(t, x)=\beta(t)$ for $t \in\left[s_{1}, s_{0}\right]$, and there exists $v \in \widetilde{S}_{F, x}$ with $v(t) \leq v_{2}(t)$ and $v(t) \in F(t, \beta(t))$ such that

$$
\begin{aligned}
\beta\left(s_{0}\right)-\beta\left(s_{1}\right) & \leq x\left(s_{0}\right)-x\left(s_{1}\right)=\int_{s_{1}}^{s_{0}}[v(s)+\beta(s)-x(s)] d s \\
& <\int_{s_{1}}^{s_{0}} v(s) d s \leq \int_{s_{1}}^{s_{0}} v_{2}(s) d s \\
& \leq \int_{s_{1}}^{s_{0}} \beta^{\prime}(s) d s=\beta\left(s_{0}\right)-\beta\left(s_{1}\right) .
\end{aligned}
$$

This is a contradiction. Consequently, $x(t) \leq \beta(t)$ for all $t \in J$.

Similarly, we can prove that $\alpha(t) \leq x(t)$ on $J$. This shows that (3.9) holds.

Finally, we prove that the solution $x$ of (3.1) satisfies (3.10). Suppose that

$$
\alpha(0)>x(0)-g(\tau(0, x), \tau(T, x)) .
$$

Then with the boundary condition of (3.1) and the definition of $\tau$, we have

$$
x(0)=\alpha(0) .
$$

With (3.9) and the definition of $\tau$, we have

$$
\tau(0, x)=x(0), \tau(T, x)=x(T)
$$

From (3.11) to (3.13), we get

$$
g(\alpha(0), x(T))=g(\tau(0, x), \tau(T, x))>0 .
$$


Since $g$ is nondecreasing in the second variable in (H4) and $x(T) \leq \beta(T)$, we have

$$
g(\alpha(0), \beta(T)) \geq g(\alpha(0), x(T))>0,
$$

which contradicts $g(\alpha(0), \beta(T)) \leq 0$ in Definition 2. So, we have

$$
\alpha(0) \leq x(0)-g(\tau(0, x), \tau(T, x)) .
$$

Analogously, we can prove that

$$
x(0)-g(\tau(0, x), \tau(T, x)) \leq \beta(0) .
$$

(3.14) and (3.15) show that (3.10) holds.

According to Steps 1 to 5, the solution $x$ of (3.1) is also a solution of (1.1). The proof is complete.

Remark 11. If $g(x(0), x(T))=x(0)+x(T))$ in (1.1), i.e., $x(0)=-x(T)$, which satisfies (H4), then (1.1) becomes an antiperiodic boundary value problem for impulsive differential inclusions.

\section{REFERENCES}

[1] B. Ahmad, J.J. Nieto, A study of impulsive fractional differential inclusions with anti-periodic boundary conditions, Fract. Diff. Calc., 2, No. 1 (2012), 1-15.

[2] D.D. Bainov, P.S. Simeonov, Impulsive differential equations: periodic solutions and applications, Longman, Harlow (1993).

[3] M. Benchohra, J.R. Graef, S.K. Ntouyas and A. Ouahab, Upper and lower solutions method for impulsive differential inclusions with nonlinear boundary conditions and variable times, Dynam. Contin. Discrete Impuls. Systems Series A: Math. Anal., 12, No-s: 3-4 (2005), 383-396.

[4] M. Benchohra, J. Henderson, S.K. Ntouyas, On first order impulsive differential inclusions with periodic boundary conditions, Dynam. Contin. Discrete Impuls. Systems Series A: Math. Anal., 9, No. 3 (2002), 417-427.

[5] E.N. Chukwu, Differential models and neutral systems for controlling the wealth of nations, Workd Scientific, River Edge (2001).

[6] L.H. Erbe, H.I. Freedman, X.Z. Liu and J.H. Wu, Comparison principles for impulsive parabolic equations with applications to models of single species growth, J. Austral. Math. Soc. Ser. B, 32 (1991), 382-400.

[7] G. Gabor, Differential inclusions with state-dependent impulses on the half-line: New Frchet space of functions and structure of solution sets, J. Math. Anal. Appl., 446, No. 2 (2017), 1427-1448. 
[8] J.K. Hale, Ordinary differential equations, Pure and Appl. Math., John Wiley and Sons, New York (1969).

[9] A. Hadjian, S. Heidarkhani, Existence of one non-trivial anti-periodic solution for second-order impulsive differential inclusions, Math. Meth. Appl. Sci., 40, No. 14 (2017), 5009-5017.

[10] S. Heidarkhani, G.A. Afrouzi, A. Hadjian, Existence of three anti-periodic solutions for second-order impulsive differential inclusions with two parameters, Discussiones Mathematicae Differential Inclusions, Control and Optimization, 33, No. 2 (2013), 115-133.

[11] S. Heidarkhani, G.A. Afrouzi, A. Hadjian, J. Henderson, Existence of infinitely many anti-periodic solutions for second-order impulsive differential inclusions, Electron. J. Diff. Equ., 2013, No. 97 (2013), 1-13.

[12] M. Kisielewicz, Differential inclusions and optimal control, Springer, Netherlands (1991).

[13] M. Kamenskii, V. Obukhovskii and P. Zecca, Condensing multivalued maps and semilinear differential inclusions in Banach spaces, Walter de Gruyter Co., Berlin, Germany (2001).

[14] A. Lasota and Z. Opial, An application of the Kakutani-Ky-Fan theorem in the theory of ordinary differential equations, Bull. Acad. Pol. Sci. Ser. Sci. Math. Astronom. Phys., 13 (1965), 781-786.

[15] M. Martelli, A Rothe's type theorem for noncompact acyclic-valued maps, Boll. Un. Mat. Ital., 4, No. 3 (1975), 70-76.

[16] N. Nyamoradi, Existence and multiplicity of solutions for second-order impulsive differential inclusions, J. Contemp. Math. Anal., 49, No. 1 (2014), 33-41.

[17] Y. Tian, J. Hendenson, Three anti-periodic solutions for second-order impulsive differential inclusions via nonsmooth critical point theory, Nonlinear Anal., 75, No. 18 (2012), 6496-6505.

[18] L.M. Wang, L.S. Chen and J.J. Nieto, The dynamics of an epidemic model for pest control with impulsive effect, Nonlinear Anal. Real World Appl., 11 (2010), 1374-1386. 